\title{
Gradhiva
}

GRADHIV

Revue d'anthropologie et d'histoire des arts

$22 \mid 2015$

Cosmos

\section{Estelle Amy de la Bretèque, Paroles mélodisées. Récits épiques et lamentations chez les Yézidis d'Arménie}

Paris, Classiques Garnier, 2013

Jean-Charles Depaule

\section{OpenEdition}

Journals

Édition électronique

URL : http://journals.openedition.org/gradhiva/3105

DOI : $10.4000 /$ gradhiva.3105

ISSN : 1760-849X

Éditeur

Musée du quai Branly Jacques Chirac

Édition imprimée

Date de publication : 1 octobre 2015

Pagination : 226-227

ISBN : 978-2-35744-092-0

ISSN : 0764-8928

Référence électronique

Jean-Charles Depaule, «Estelle Amy de la Bretèque, Paroles mélodisées. Récits épiques et lamentations chez les Yézidis d'Arménie », Gradhiva [En ligne], 22 | 2015, mis en ligne le 18 septembre 2015, consulté le 24 septembre 2020. URL : http://journals.openedition.org/gradhiva/3105 ; DOI : https://doi.org/ 10.4000/gradhiva.3105

Ce document a été généré automatiquement le 24 septembre 2020

(c) musée du quai Branly 
Estelle Amy de la Bretèque, Paroles mélodisées. Récits épiques et lamentations chez les Yézidis d'Arménie

Paris, Classiques Garnier, 2013

Jean-Charles Depaule

\section{RÉFÉRENCE}

Estelle Amy de la Bretèque, Paroles mélodisées. Récits épiques et lamentations chez les Yézidis d'Arménie. Paris, Classiques Garnier, 2013, 230 p. 
Estelle Amy de la Bretèque

\section{Paroles mélodisées}

Récits épiques et lamentations chez les Yézidis d'Arménie

la communauté yézidie d'Irak lorsque, en août 2014, après la prise de Sinjar, des milliers de ses membres ont été pourchassés par les troupes de Daesh. L'histoire des Yézidis, qui sont kurdophones, a été ponctuée à travers les siècles par des persécutions et des exodes. Souvent accusés d'être des adorateurs du Diable, ils pratiquent un monothéisme syncrétique. Ils ne font pas partie des gens du Livre auxquels l'islam confère en principe un statut de "protégés ». On naît yézidi (on ne le devient pas) dans une société structurée en groupes endogames.

1 Si la majorité vit en Irak, où se trouvent, au nord, le principal lieu de pèlerinage et le siège de leur chef religieux, des Yézidis, originaires d'Anatolie, se sont établis au XIX siècle, puis au début du $\mathrm{xx}^{\mathrm{e}}$ siècle, en face du mythique mont Ararat. Estelle Amy de la Bretèque, ethnomusicologue, s'est intéressée à cette communauté où l'on parle généralement kurde et arménien, éventuellement russe. Les groupes endogames y sont au nombre de trois. Les deux premiers sont ceux des religieux dont les fonctions rituelles se différencient peu. Les "disciples", qui ne connaissent pas les poèmes sacrés, forment le troisième groupe et se définissent par le fait de n'être pas nés dans une famille de religieux. Chacun est lié personnellement à un membre des deux autres groupes dans une relation d'obligations réciproques.

2 "L'art d'orienter les antennes paraboliques» (ou spoutnik) - c'est le titre d'un des chapitres introductifs - est significatif de la manière dont les Yézidis d'Arménie «composent» une identité problématique, souvent suspecte pour leurs voisins polémique, écrit l'auteure -, qu'il s'agisse de la langue ou de la politique selon leur degré d'adhésion à la cause kurde ou d'intégration au contexte arménien. Et de leur rapport à la musique, en particulier au rabiz, genre post-soviétique " pop » qu'on écoute en ville, mais non dans les villages, et qui compte des vedettes parmi les musiciens yézidis chantant, non seulement en arménien et en kurde, mais parfois en géorgien ou en russe. Spoutnik et rabiz, note l'auteure, suggèrent "un espace et un temps 
charnières; entre les empires d'hier et de demain, entre le monde soviétique et le néocapitalisme, entre les chrétiens et les musulmans ».

Sur les marchés d'Erevan ou des grandes villes de Russie, on trouve des compilations contenant quelques morceaux, généralement enregistrés en studio, commémorant des figures yézidies exemplaires, des soldats tués sur le front du Karabagh, un mafieux assassiné... Estelle Amy de la Bretèque a plus spécialement étudié des lamentations funèbres, qui mobilisent les mêmes procédés formels que ces célébrations de «héros d'hier ou d'aujourd'hui » au destin tragique. Elle s'est s'attachée à la façon dont, dans des contextes rituels ou au cours d'une banale conversation, des « cœurs brûlants », le plus souvent des femmes impliquées dans un deuil, éternellement endeuillées, ou des professionnels sans doute plus distants «mélodisent » des kilamê ser, " paroles sur ». En s'appuyant sur le riche corpus qu'elle a recueilli au cours des années 2000 dans le cadre d'un travail de thèse et dont de nombreux extraits sont consultables en ligne, elle montre en quoi consiste ce genre d'énonciation bien caractérisé dont elle a analysé les propriétés prosodiques.

4 Ce n'est pas le "parlé chanté ", sprechgesang, tel que l'a défini Schönberg, mais plutôt une sorte de récitatif, de déclamation ou cantillation, d'où l'emploi du néologisme «mélodiser». Les Yézidis le distinguent du chant, le stran, une forme vocale ou instrumentale jouée lors de fêtes calendaires, notamment carnavalesques. Il est lié à la danse et sa pulsation est régulière, à la différence des paroles mélodisées qui appartiennent à une temporalité favorisant l'écoute. L'opposition est donc nette, le chant est associé à la joie et à la danse, et la parole mélodisée, à la tristesse. Le zurna, hautbois, et le dohol, tambour sur cadre, accompagnent le stran. Intervenant souvent par paire, l'un dessinant la ligne mélodique tandis que l'autre tient le bourdon, le duduk, un hautbois plus grave que le zurna (qui comme lui est répandu dans tout le Moyen-Orient), est réservé aux kilamê ser. Son jeu est lui-même nommé " parole sur ", il raconte des choses tristes.

5 Les paroles que mélodisent «les voix multiples» des énonciatrices et d'éventuels professionnels, les sanglots et les plaintes qu'elles suscitent dans l'assistance tissent un réseau qui, en impliquant la famille et l'auditoire, «crée un espace commun, hors de la réalité tangible », un " espace de peine partagé ». Elles entretiennent la souffrance. Ce que les kilamê ser ne cessent de rappeler, c'est qu'on ne guérit pas du deuil, on doit vivre avec lui. Il faut se consacrer à la mémoire de celui qui est mort en mélodisant dans les fêtes et dans la vie de tous les jours, lors d'une visite amicale, dans une cuisine aussi bien (avec de surprenants changements de registre, des passages sans transition du parléparlé au parlé-mélodisé et vice versa). Les paroles sont sous-tendues par le qurban - ce mot d'origine arabe désigne un sacrifice rituel, mais il peut dans le parler quotidien être investi d'une grande tendresse : pour une mère son enfant est son qurban, son beau souci. Elles sont nourries par le sentiment constant de l'exil, lui-même nourri d'exils divers qui, dans les "paroles sur", sont souvent mêlés, l'exode collectif que la communauté a connu, l'éloignement de proches qui sont partis chercher du travail en Russie, en Allemagne, le départ du foyer paternel d'une fille qui se marie. Et la mort, bien sûr. Lors de l'enterrement de Yurîk, un jeune homme poignardé, en Allemagne, par un Arménien, une des femmes au cœur brûlant dit - le défunt parle par sa bouche : un chemin s'est ouvert devant moi, je connais l'aller mais pas le retour. D'où l'importance des lieux mentionnés par les « paroles sur » qui, de l'endroit où la neige fond ou de la porte 
de la maison de ton frère où un corbeau croasse à Moscou la maudite, forment ce que l'auteure nomme une " topographie émotionnelle».

6 Selon Estelle Amy de la Bretèque, qui cite en outre plusieurs «traditions lamentées " comparables étudiées à travers le monde par les ethnologues, ces manifestations dépassent les limites de la communauté yézidie d'Arménie qui s'insère, à cet égard, dans « une culture régionale de la douleur et de l'exil». Cette culture, précise-t-elle, n'est pas seulement caucasienne, elle «est présente historiquement en Anatolie dans les communautés kurdophones, arménophones, grécophones et turcophones ».

L'analyse des conditions d'une telle coproduction d'émotions est soutenue par l'observation attentive du fonctionnement rhétorique de la parole et de la gestuelle (les signes partagés de l'affliction sont inventoriés). Et par l'identification des références et formules partagées, obligées pourrait-on dire, les fleurs sur les cimes, les roses de Bagdad... qui, entre terrain familier et découverte, sollicitent l'auditeur. L'auteure souligne la présence récurrente des mêmes interjections, spécialement wey le mine, et du discours rapporté (je dis, il dit, il a dit, ils ont dit...) dont l'usage est courant dans les propos quotidiens.

8 Elle souligne également la manière dont les événements évoqués sont situés dans une temporalité flottante et dont les "paroles » se déploient au-delà des frontières d'une expression strictement langagière. Elle explicite lumineusement ce dernier aspect en revenant sur la fonction du hautbois qui, on l'a vu, parle lui aussi : "Sans sémantisme clair, la parole du duduk peut [...] être rapprochée des interjections. Cette comparaison, couramment exprimée par la phrase "Le duduk dit wey le mine", présente le duduk comme une abstraction du vocal... » À moins, ajoute-t-elle, de percevoir, à l'inverse, ces interjections comme les substituts de l'instrument. «Les commentaires des Yézidis sur le jeu du duduk montrent en tout cas que pour dire des "paroles sur", il n'est pas nécessaire de les énoncer verbalement. »

Enfin, Estelle Amy de la Bretèque, en citant Bakhtine, propose un rapprochement entre l'emploi du discours rapporté et de la mélodisation et notre usage des guillemets qui détachent ce qu'ils mettent en exergue. Émotion et distance. Il me semble que ces procédés formels font accéder les cœurs brûlants et leur auditoire à un registre épique qui n'est pas réservé aux héros labellisés. Je pense à Brecht s'interrogeant sur son travail de dramaturge. L'auteure apporte ainsi une intéressante contribution à une science plus générale du poétique, de la fonction poétique ou poéticité, comme aurait dit Jakobson, englobant les expressions populaires et savantes, improvisées ou non, y compris la poésie dite sonore, ou même bruitiste.

10 Une dernière remarque, marginale peut-être : la transcription des paroles mélodisées, respectant comme il se doit interjections, répétitions et propositions indécidables, est accompagnée d'une traduction souvent littérale dont l'efficacité et le pouvoir de suggestion sont remarquables. On rejoint ici la réflexion en acte des traducteurs de poésie contemporains partagés entre, d'une part, la volonté de traduire un texte en l'acclimatant au français, à sa rhétorique et à sa tradition prosodique, voire d'en faire un poème de chez nous, et, d'autre part, un parti plus radical (de littéralité), le souhait en tout cas de faire entendre la langue d'origine dans la nôtre.

11 On l'aura compris, Estelle Amy de la Bretèque a réussi à rendre compte avec une grande précision de l'objet auquel elle s'est attachée, sans rien sacrifier de sa complexité, de sa 
labilité, de ses subtilités et de l'émotion dont il déborde. Son livre est un modèle du genre.

\section{AUTEURS}

\section{JEAN-CHARLES DEPAULE}

jean-charles.depaule898@orange.fr 